\title{
Opinion Dynamics with Bayesian Learning
}

\author{
Aili Fang $\mathbb{D}^{1},{ }^{1}$ Kehua Yuan, ${ }^{2}$ Jinhua Geng, ${ }^{3}$ and Xinjiang Wei ${ }^{1}$ \\ ${ }^{1}$ School of Mathematics and Statistics, Ludong University, Yantai 264025, China \\ ${ }^{2}$ School of Mathematics and Statistics, Southwest University, Chongqing 400715, China \\ ${ }^{3}$ Institute of Complexity Science, Qingdao University, Qingdao 266071, China \\ Correspondence should be addressed to Aili Fang; fangaili@hotmail.com
}

Received 3 November 2019; Revised 16 January 2020; Accepted 21 January 2020; Published 22 February 2020

Academic Editor: Yongping Pan

Copyright (c) 2020 Aili Fang et al. This is an open access article distributed under the Creative Commons Attribution License, which permits unrestricted use, distribution, and reproduction in any medium, provided the original work is properly cited.

\begin{abstract}
Bayesian learning is a rational and effective strategy in the opinion dynamic process. In this paper, we theoretically prove that individual Bayesian learning can realize asymptotic learning and we test it by simulations on the Zachary network. Then, we propose a Bayesian social learning model with signal update strategy and apply the model on the Zachary network to observe opinion dynamics. Finally, we contrast the two learning strategies and find that Bayesian social learning can lead to asymptotic learning more faster than individual Bayesian learning.
\end{abstract}

\section{Introduction}

We all have our own opinions on various topics of social issues. The opinions are formed by an evolutionary process in social context [1-3]. Opinion dynamics is the study of the opinion fusion process through interactions among a group of agents [4]. Some interesting models have been proposed, such as the DeGroot model [5], voter model [6, 7], bounded confidence model [8-10], and many other models [11-14]. Among these models, a key element is the opinion update strategy in the dynamic process. All the opinion update strategies can be classified into two categories by using Bayesian update rule or not: one is Bayesian update strategy and the other is nonBayesian strategy. Non-Bayesian learning refers to individuals updating their opinions by non-Bayesian strategy, such as linear combination of opinions of the neighbors $[5,15,16]$ and various game theories in social network $[17,18]$. Most of these models try to explore how the society could achieve group consensus. But the consensus opinion might not necessarily be the truth; in other words, the true state might not be realized. While Bayesian learning assumes that individual updates their opinions according to Bayesian rule, individuals can learn the truth in the long run according to the prior information [19-21]. Therefore, Bayesian learning can integrate the prior information and lead to realize the truth more rationally and effectively than non-Bayesian learning.
Though Bayesian learning can achieve the truth, there is no systematical proof in the existing literature $[22,23]$. The proof of individual Bayesian learning lays a theoretical foundation for the learning process, so in this paper, we will deduce the truthfulness of individual Bayesian learning by theoretical derivation. Moreover, considering people's crowd psychology, we put forward a signal update strategy which means that people adjust their observations to meet with the most people. Combined with this signal update strategy, we propose a Bayesian social learning model to study the opinion dynamics under social environment. Furthermore, we conduct simulations on Zachary network to observe the learning results.

The rest of the paper is organized as follows. In Section 2, we give a theoretical proof of individual Bayesian learning. In Section 3, we propose a Bayesian social learning model with signal update strategy and test it by simulations on Zachary network. In Section 4, we draw conclusions.

\section{Individual Bayesian Learning}

2.1. Individual Bayesian Learning Model. Let state space $\Theta=$ $\left\{\theta_{1}, \theta_{2}, \ldots, \theta_{m}\right\}$ and the underlying true state be $\theta^{*}\left(\theta^{*} \in \Theta\right)$. Individual $i$ 's opinion on state $\theta_{k}$ at time $t$ is denoted by 
probability distribution $\mu_{i, t}\left(\theta_{k}\right) k=1,2, \ldots, m$, where $m$ is a finite integer. At each time period, the signal set $S_{t}=\left\{S_{t}^{1}, S_{t}^{2}, \ldots, S_{t}^{n}\right\} \in S_{1} \times \cdots \times S_{n}$ is generated by the likelihood function $P\left(S_{t} \mid \theta_{k}\right)$ conditional on state $\theta_{k}$, where $S_{t}^{i} \in S^{i}$ denotes the signal privately observed by individual $i$ at time $t$ and $S^{i}$ denotes the individual $i$ 's signal space. The $i$ th marginal of $P\left(\theta_{k}\right)$ is denoted by $P_{i}\left(\theta_{k}\right)$ which is known as individual is signal structure conditional on state $\theta_{k}$. We assume that each individual's private signal structure is commonly known. At each time, individual $i$ receives his private signal $S_{t}^{i}$ and updates his prior opinion to the posterior opinion by Bayesian law. Bayesian statistics combining prior information can make the inference results more accurate and effective [24]. As time goes by, the individual's opinion will show some amazing dynamics during the evolution process. Next, we will give the denotations of asymptotic learning which is an important definition in opinion dynamics.

Definition 1. Asymptotic learning: asymptotic learning refers to that individual $i$ who receives the signals generated by true state $\theta^{*} \in \Theta$ and achieves asymptotic learning on a path $\left\{S_{t}^{i}\right\}_{\mathrm{t}=1}^{\infty}$ if along that path $\mu_{i, t}\left(\theta^{*}\right) \longrightarrow 1$ with probability one as $\mathrm{t} \longrightarrow \infty$.

\subsection{Theorem and Proof}

\section{Theorem 1. Assume that}

(1) Individual $i$ has positive prior belief on true state $\theta^{*}$, i.e., $\mu_{i, 0}\left(\theta^{*}\right)>0$.

(2) At each time $t, \forall S_{t}^{i} \in S_{t}, P\left(S_{t}^{i} \mid \theta\right)>0$ holds for all $i$ and $\theta$.

(3) There is no observationally equivalent state to $\theta^{*}$ for individual i, i.e., $\left\{\theta \in \Theta \mid P_{i}(s \mid \theta)=P_{i}\left(s \mid \theta^{*}\right)\right.$, $\left.\forall s \in S_{i}\right\}=\varnothing$.

At each time step $t$, individual $i$ has his prior opinion $\mu_{i, t}(\theta)$ on state $\theta$; after receiving signal $S_{t+1}^{i}$ which is generated by true state $\theta^{*} \in \Theta$, his opinion $\mu_{i, t+1}(\theta)$ at the next time step $t+1$ will be updated to his posterior probability by Bayesian law:

$$
\begin{aligned}
\mu_{i, t+1}(\theta) & =\mu_{i, t+1}\left(\theta \mid\left(S_{1}^{i}, S_{2}^{i}, \ldots, S_{t}^{i}, S_{t+1}^{i}\right)\right) \\
& =\frac{\mu_{i, t}(\theta) P\left(\left(S_{1}^{i}, S_{2}^{i}, \ldots, S_{t}^{i}, S_{t+1}^{i}\right) \mid \theta\right)}{P\left(S_{1}^{i}, S_{2}^{i}, \ldots, S_{t}^{i}, S_{t+1}^{i}\right)}(\theta \in \Theta) .
\end{aligned}
$$

Then, $\mu_{i, t}\left(\theta^{*}\right) \longrightarrow 1$ will hold with probability one as $t \longrightarrow \infty$ and we call individual $i$ realizes asymptotic learning.

Proof. Let state space $\Theta=\left\{\theta_{1}, \theta_{2}, \ldots, \theta_{m}\right\}$ and the true state $\theta^{*}=\theta_{1}$. Suppose that the signal sequences $S_{t}^{i}(t=1,2, \ldots)$ are independent and identically distributed given $\theta_{k}$, then we have

$$
P\left(\left(S_{1}^{i}, S_{2}^{i}, \ldots, S_{t}^{i}\right) \mid \theta_{k}\right)=\prod_{r=1}^{t} P\left(S_{r}^{i} \mid \theta_{k}\right)
$$

At time $t+1$, individual $i$ updates his opinion and obtains his posterior opinion by Bayesian rule (1) after receiving signal $S_{t+1}^{i}$.

$$
\begin{aligned}
\mu_{i, t+1}\left(\theta_{k}\right) & =\mu_{i, t+1}\left(\theta_{k} \mid\left(S_{1}^{i}, S_{2}^{i}, \ldots, S_{t}^{i}, S_{t+1}^{i}\right)\right) \\
& =\frac{\mu_{i, t}\left(\theta_{k}\right) P\left(\left(S_{1}^{i}, S_{2}^{i}, \ldots, S_{t}^{i}, S_{t+1}^{i}\right) \mid \theta_{k}\right)}{P\left(S_{1}^{i}, S_{2}^{i}, \ldots, S_{t}^{i}, S_{t+1}^{i}\right)} \\
& =\mu_{i, t}\left(\theta_{k}\right) \frac{\prod_{r=1}^{t+1} P\left(S_{r}^{i} \mid \theta_{k}\right)}{\sum_{\theta \epsilon \Theta} \mu_{i, t}(\theta) \prod_{r=1}^{t+1} P\left(S_{r}^{i} \mid \theta\right) .}
\end{aligned}
$$

Next, we compare the probability of two state of the partition, say $\theta_{1}$ and $\theta_{2}$ in the light of $S_{t+1}^{i}$ for individual $i$ at time $t+1$,

$$
\begin{aligned}
\frac{\mu_{i, t+1}\left(\theta_{1}\right)}{\mu_{i, t+1}\left(\theta_{2}\right)} & =\frac{\mu_{i, t}\left(\theta_{1}\right)}{\mu_{i, t}\left(\theta_{2}\right)} \prod_{r=1}^{t+1} \frac{P\left(S_{r}^{i} \mid \theta_{1}\right)}{P\left(S_{r}^{i} \mid \theta_{2}\right)} \\
& =\frac{\mu_{i, t}\left(\theta_{1}\right)}{\mu_{i, t}\left(\theta_{2}\right)} \prod_{r=1}^{t+1} \delta\left(S_{r}^{i}\right) \\
& =\frac{\mu_{i, t}\left(\theta_{1}\right)}{\mu_{i, t}\left(\theta_{2}\right)} R\left(S_{t+1}^{i}\right),
\end{aligned}
$$

where $\delta\left(S_{r}^{i}\right)$ is the likelihood ration of $\theta_{1}$ to $\theta_{2}$ given $S_{r}^{i}$ and $R\left(S_{t+1}^{i}\right)$ is the likelihood ration of $\theta_{1}$ to $\theta_{2}$ given $\left\{S_{1}^{i}, S_{2}^{i}, \ldots, S_{t+1}^{i}\right\}$.

Suppose $P\left(\delta\left(S_{r}^{i}\right)<\infty \mid \theta_{1}\right)=\phi$, two cases are to be distinguished.

In the first case, suppose $\phi<1$, then $P\left(R\left(S_{t}^{i}\right)=\infty \mid \theta_{1}\right)=$ $1-\phi^{t}$ which obviously approaches 1 with increasing $t$. Another forms of expression can be

$$
\lim _{t \longrightarrow \infty} P\left(R\left(S_{t}^{i}\right) \geq \rho \mid \theta_{1}\right)=1 \text { for } \quad 0 \leq \rho<\infty .
$$

Equation (5) shows that the probability that $\operatorname{PR}\left(S_{t}^{i}\right)$ given $\theta_{1}$ is greater than any preassigned number is almost 1 .

The second case is $\phi=1$. Since much is known about sums of identically distributed independent random variables, it is natural to investigate

$$
\log R\left(S_{t+1}^{i}\right)=\sum_{r=1}^{t+1} \log \delta\left(S_{r}^{i}\right),
$$

thereby replacing a product by a sum. It is easily seen from the definition of $\delta\left(S_{r}^{i}\right)$ that $\delta\left(S_{r}^{i}>0 \mid \theta_{1}\right)=1$, so for the case now at hand, the function $\log \left(\delta\left(S_{r}^{i}\right)\right)$ is independent real bounded random variable.

Letting $I=E\left(\log \left(\delta\left(S_{r}^{i}\right)\right) \mid \theta_{1}\right)$, the weak law of large numbers implies that, for any $\varepsilon>0$,

$$
\begin{gathered}
\lim _{t \rightarrow \infty} P\left(\mid \frac{1}{t+1} \sum_{i=1}^{t+1}\left[\log \left(R\left(S_{t+1}^{i}\right)\right)\right.\right. \\
\left.\left.-E\left(\log \left(R\left(S_{t+1}^{i}\right)\right)\right)\right]|\leq \varepsilon| \theta_{1}\right)=1,
\end{gathered}
$$

and it can be transformed into 


$$
\lim _{t \longrightarrow \infty} P\left(\log \left(R\left(S_{t+1}^{i}\right) \geq(t+1)(I-\varepsilon) \mid \theta_{1}\right)\right)=1 .
$$

Equivalently,

$$
\lim _{t \longrightarrow \infty} P\left(R\left(S_{t+1}^{i}\right) \geq e^{(t+1)(I-\varepsilon)} \mid \theta_{1}\right)=1 .
$$

According to expectation inequality [25], consider that

$$
\begin{aligned}
I & =E\left(\log \left(\delta\left(S_{r}^{i}\right)\right) \mid \theta_{1}\right) \\
& \geq \log E^{-1}\left(\delta^{-1}\left(S_{r}^{i}\right) \mid \theta_{1}\right) \\
& =-\log E\left(\frac{P\left(S_{r}^{i} \mid \theta_{2}\right)}{P\left(S_{r}^{i} \mid \theta_{1}\right)} \mid \theta_{1}\right) \\
& =-\log \sum_{S_{r}^{i} \in S_{t+1}^{i}} \frac{P\left(S_{r}^{i} \mid \theta_{2}\right)}{P\left(S_{r}^{i} \mid \theta_{1}\right)} \cdot P\left(S_{r}^{i} \mid \theta_{1}\right) \\
& =-\log \sum_{S_{r}^{i} \in S_{t+1}^{i}} P\left(S_{r}^{i} \mid \theta_{2}\right) \\
& =-\log 1=0,
\end{aligned}
$$

the equality can hold in (10) if and only if $\delta^{-1}\left(S_{r}^{i}\right)$ is constant with probability 1 , given $\theta_{1}$. Since the expected value of $\delta^{-1}\left(S_{r}^{i}\right)$ is equal to 1 , the equality will hold if and only if $P\left(\delta^{-1}\left(S_{r}^{i}\right) \mid \theta_{1}\right)=1$. This means state $\theta_{2}$ is observationally equivalent state to $\theta_{1}$, which is contradictory with condition (3) of the theorem. So, $I>0$. Then, according to (9), we can also infer that (5) holds.

Therefore, under the assumption conditions of the theorem, we can demonstrate $P\left(R\left(S_{t}^{i}\right)=\infty \mid \theta_{1}\right)=1$ holds with probability one; consequently, according to (10), the ratio of individual is posterior probability of the real state $\theta^{*}=\theta_{1}$ to the other state $\theta_{2}$ tends to infinity, i.e.,

$$
\frac{\mu_{i, t+1}\left(\theta^{*}\right)}{\mu_{i, t+1}\left(\theta_{2}\right)} \longrightarrow \infty
$$

By the same method, it can be proved that the likelihood ratio functions of real states $\theta^{*}$ to the other states $\theta_{k}, k=$ $3,4, \ldots, m$ also tend to infinity, i.e.,

$$
\frac{\mu_{i, t+1}\left(\theta^{*}\right)}{\mu_{i, t+1}\left(\theta_{k}\right)} \longrightarrow \infty
$$

Since $\mu_{i, t+1}\left(\theta^{*}\right)+\sum_{i=2}^{m} \mu_{i, t+1}\left(\theta_{k}\right)=1$ and $0 \leq \mu_{i, t+1}(\theta)$ $\leq 1(\forall \theta \in \Theta)$, we will have $\mu_{i, t+1}\left(\theta^{*}\right) \longrightarrow 1$ and $\mu_{i, t+1}\left(\theta_{k}\right) \longrightarrow 0\left(\forall \theta_{k} \neq \theta^{*}\right)$ when $t \longrightarrow \infty$.

In summary, the individual can become highly convinced of the truth by the Bayesian law and achieve asymptotic learning after abundant observations.

2.3. Simulation. We have theoretically proved that individuals can use Bayesian law to update their opinions and achieve asymptotic learning. Next, we will verify the truth of the above individual Bayesian learning model by simulations on individuals in Zachary network.

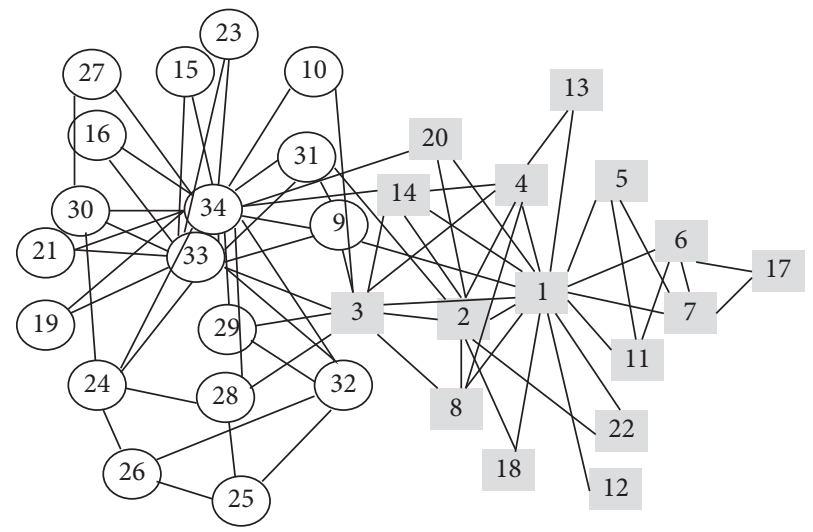

FIGURE 1: Zachary network.

2.3.1. Initial Values Setting. Before conducting the numerical simulations, some assumptions are given as follows:

(1) Different signals are independent of each other

(2) The connection between individuals is indirect

(3) In the initial state, all individuals have the same opinion in different states

(4) Each individual observes only one signal at a time

(5) Different individuals have the same signal structure

This simulation experiment mainly aims at social learning on two states. Now, we suppose that $\Theta=\left\{\theta_{1}, \theta_{2}\right\}$ and the true state $\theta^{*}=\theta_{1}$, and the signal space $S=\left\{S_{1}, S_{2}, \ldots, S_{5}\right\}$. The individual composition and their relationship are shown in Figure 1, which is famously known as Zachary network [26].

At time $t=0$, individual $i$ 's opinion on $\theta_{1}$ and $\theta_{2}$ is $\mu_{i, 0}\left(\theta_{1}\right)$ and $\mu_{i, 0}\left(\theta_{2}\right)$, respectively. Under assumption (3), the individuals' initial opinions are set to be

$$
\begin{aligned}
& \left\{\mu_{1,0}\left(\theta_{1}\right), \mu_{2,0}\left(\theta_{1}\right), \ldots, \mu_{34,0}\left(\theta_{1}\right)\right\}=\{0.5,0.5, \ldots, 0.5\}, \\
& \left\{\mu_{1,0}\left(\theta_{2}\right), \mu_{2,0}\left(\theta_{2}\right), \ldots, \mu_{34,0}\left(\theta_{2}\right)\right\}=\{0.5,0.5, \ldots, 0.5\} .
\end{aligned}
$$

Under assumption (5), the signal structures are given for all $i$ as follows and they will remain unchanged during the learning process:

$$
\begin{array}{r}
\left\{P_{i}\left(S_{1} \mid \theta_{1}\right), P_{i}\left(S_{2} \mid \theta_{1}\right), \ldots, P_{i}\left(S_{5} \mid \theta_{1}\right)\right\} \\
=\{0.35,0.20,0.15,0.05,0.25\}, \\
\left\{P_{i}\left(S_{1} \mid \theta_{2}\right), P_{i}\left(S_{2} \mid \theta_{2}\right), \ldots, P_{i}\left(S_{5} \mid \theta_{2}\right)\right\} \\
=\{0.30,0.15,0.10,0.25,0.20\} .
\end{array}
$$

In the individual Bayesian learning model, it is assumed that individual opinion evolution is affected by their prior knowledge and signal characteristics. When the individual $i$ 's opinion on the underlying true state $\mu_{i, t}\left(\theta^{*}\right)$ is larger than 0.9999 , we consider that he reaches asymptotic learning. If each individual realizes asymptotic learning, the whole society will form a social consensus and find the truth. 


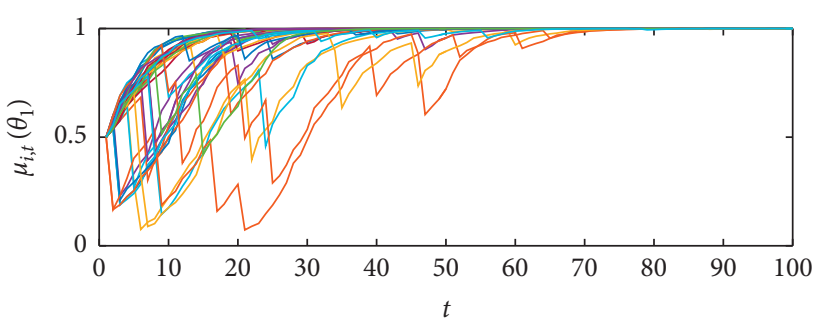

(a)

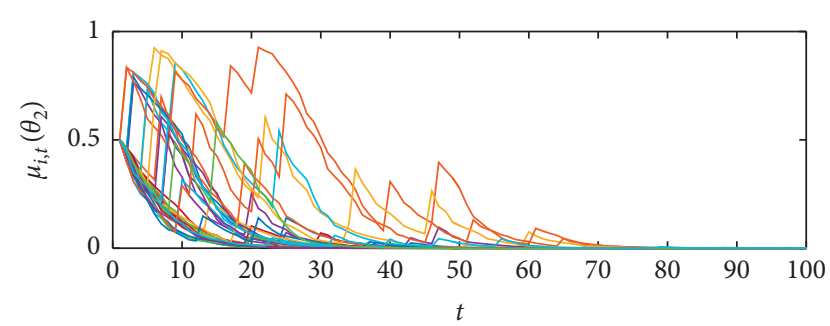

(b)

Figure 2: Individual Bayesian learning results.

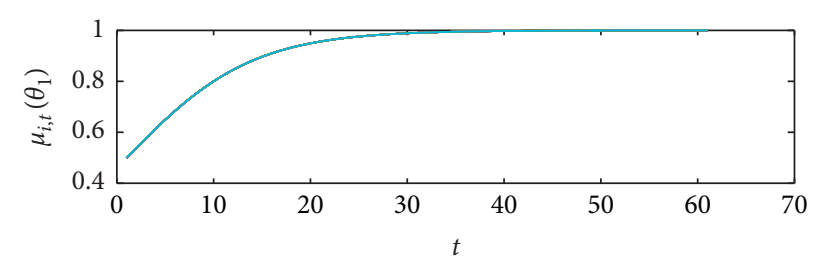

(a)

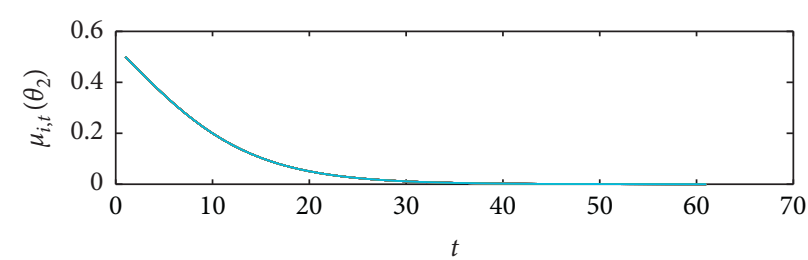

(b)

FIGURE 3: Bayesian social learning results.

2.3.2. Simulation Results. Under the above initial conditions, we conduct simulations on Zachary network, and results in Figure 2 show that $\mu_{i, t}\left(\theta_{1}\right) \longrightarrow 1$ and $\mu_{i, t}\left(\theta_{2}\right) \longrightarrow 0$ when $t \longrightarrow \infty$ for all $i$, which means all the individuals can achieve asymptotic learning and realize the true state.

\section{Bayesian Social Learning Model}

3.1. Bayesian Social Learning. In the individual Bayesian learning process, individuals update their opinions by Bayesian law to achieve asymptotic learning. But the whole society is a complex social network, and individuals are connected and have influences on each other. By communication with his neighbors, the individual can have knowledge of his neighbors' received signals and their opinions. So, the individual will continuously adjust his opinion according to the signals received not only by himself but also by his neighbors. Therefore, we consider opinion dynamics with Bayesian law in a social network background, which is called Bayesian social learning.

Here, the social network is abstracted into a graph $G=$ $(V, E)$ composed of individuals and their interactions. Let $V$ represent the set of all individuals and $E$ represent the set of relationship between every two individuals. At time $t+1$, each individual $i$ receives a signal $S_{t+1}^{i}$ whose distribution follows his signal structure $P_{i}\left(\cdot \mid \theta^{*}\right)$. Because the individuals have influence on each other and people have herd mentality, then the individual $i$ will adjust his signal to the signal $\widehat{S}_{t+1}$ which is received by the most people. Therefore, individuals update their signals by the following rule:

$$
S_{t+1}^{i}= \begin{cases}S_{t+1}^{i}, & \text { if } S_{t+1}^{i}=\widehat{S}_{t+1} \\ \widehat{S}_{t+1}, & \text { if } S_{t+1}^{i} \neq \widehat{S}_{t+1}\end{cases}
$$

TABle 1: Comparison of average consensus time.

\begin{tabular}{lc}
\hline Model & Consensus time \\
\hline Individual Bayesian learning model & 98 \\
Bayesian social learning model & 61 \\
\hline
\end{tabular}

After updating signals, individual $i$ will update his own opinion about state $\theta$ by Bayesian law as follows:

$$
\begin{aligned}
\mu_{i, t+1}(\theta) & =\mu_{i, t+1}\left(\theta \mid\left(S_{1}^{i}, S_{2}^{i}, \ldots, S_{t}^{i}, S_{t+1}^{i}\right)\right) \\
& =\frac{\mu_{i, t}(\theta) P\left(\left(S_{1}^{i}, S_{2}^{i}, \ldots, S_{t}^{i}, S_{t+1}^{i}\right) \mid \theta\right)}{P\left(S_{1}^{i}, S_{2}^{i}, \ldots, S_{t}^{i}, S_{t+1}^{i}\right)}(\theta \in \Theta) .
\end{aligned}
$$

Next, we will explore the social learning results in the opinion dynamic process according to the signal update rule (15) and opinion update rule (16) by simulations.

3.2. Simulation Results. We also take Zachary network as an example society. The state space $\Theta=\left\{\theta_{1}, \theta_{1}\right\}$, and signal structure and initial opinions $\mu_{i, 0}(\theta)$ are set to be the same as the previous simulations. The Bayesian social learning results are shown in Figure 3.

It is obviously seen that individuals can realize asymptotic learning by Bayesian social learning with signal update strategy. The strong connectivity of the network helps the group in the network to reach social consensus quickly with little fluctuation. Furthermore, we compare the average consensus time of Bayesian social learning and individual Bayesian learning as shown in Table 1.

We can see that Bayesian social learning with the signal update strategy model has a significantly faster learning speed than the individual Bayesian learning model, and it has less fluctuation. So, we can speculate that the interactions 
among individuals in the society might accelerate information congregation and achieve asymptotic learning faster.

\section{Conclusion}

In this paper, we research on two Bayesian learning models. The first model is the individual Bayesian learning model in which we deduce the truthfulness of individual Bayesian learning by theoretical derivation. Moreover, the numerical simulations also show that individuals who update their opinions by Bayesian law can realize asymptotic learning.

Furthermore, we propose the Bayesian social learning model with signal update strategy and test it by simulations on Zachary network. The results show that individuals adopting the signal update strategy proposed in this paper can realize asymptotic learning similarly.

We compare the results of two models and find that Bayesian social learning model can achieve asymptotic learning more faster under the same conditions. In the future study, we will explore the theoretical supports for the Bayesian social learning model.

\section{Data Availability}

No data were used to support this study.

\section{Conflicts of Interest}

The authors declare that there are no conflicts of interest regarding the publication of this paper.

\section{Acknowledgments}

This work was supported by the National Natural Science Foundation of China under grant no. 61973149.

\section{References}

[1] D. Acemoglu and A. Ozdaglar, "Opinion dynamics and learning in social networks," Dynamic Games and Applications, vol. 1, no. 1, pp. 3-49, 2011.

[2] M. O. Jackson, Social and Economic Networks, Princeton University Press, Princeton, NJ, USA, 2008.

[3] J. Peng, M. T. Anahita, E. F. Noah, and B. Francesco, "Opinion dynamics and the evolution of social power in influence networks," SIAM Review, vol. 57, no. 1, pp. 367-397, 2015.

[4] Y. Dong, M. Zhan, G. Kou, Z. Ding, and H. Liang, "A survey on the fusion process in opinion dynamics," Information Fusion, vol. 43, pp. 57-65, 2018.

[5] M. H. Degroot, "Reaching a consensus," Journal of the American Statistical Association, vol. 69, no. 345, pp. 118-121, 1974.

[6] P. Clifford and A. Sudbury, "A model for spatial conflict," Biometrika, vol. 60, no. 3, pp. 581-588, 1973.

[7] R. A. Holley and T. M. Liggett, "Ergodic theorems for weakly interacting infinite systems and the voter model," The Annals of Probability, vol. 3, no. 4, pp. 643-663, 1975.

[8] R. Hegselmann and U. Krause, "Opinion dynamics and bounded confidence: models, analysis and simulation," Journal of Artificial Societies and Social Simulation, vol. 5, no. 3, 2002.
[9] G. Deffuant, D. Neau, F. Amblard, and G. Weisbuch, "Mixing beliefs among interacting agents," Advances in Complex Systems, vol. 3, no. 01n04, pp. 87-98, 2000.

[10] Q. Zha, H. Liang, G. Kou, Y. Dong, and S. Yu, "A feedback mechanism with bounded confidence-based optimization approach for consensus reaching in multiple attribute largescale group decision-making," IEEE Transactions on Computational Social Systems, vol. 6, no. 5, pp. 994-1006, 2019.

[11] S. Galam, "Minority opinion spreading in random geometry," The European Physical Journal B, vol. 25, no. 4, pp. 403-406, 2002.

[12] Y. Dong, Z. Ding, L. Martínez, and F. Herrera, "Managing consensus based on leadership in opinion dynamics," Information Sciences, vol. 397-398, pp. 187-205, 2017.

[13] A. C. R. Martins, "Continuous opinions and discrete actions in opinion dynamics problems," International Journal of Modern Physics C, vol. 19, no. 4, pp. 617-624, 2008.

[14] A. Fang, L. Wang, and X. Wei, "Social learning with multiple true states," Physica A: Statistical Mechanics and Its Applications, vol. 521, pp. 375-386, 2019.

[15] B. Golub and M. O. Jackson, "Naïve learning in social networks and the wisdom of crowds," American Economic Journal: Microeconomics, vol. 2, no. 1, pp. 112-149, 2010.

[16] P. M. Demarzo, D. Vayanos, and J. Zwiebel, "Persuasion bias, social influence, and unidimensional opinions," The Quarterly Journal of Economics, vol. 118, no. 3, pp. 909-968, 2003.

[17] D. Bauso, R. Pesenti, and M. Tolotti, "Opinion dynamics and stubbornness via multi-population mean-field games," Journal of Optimization Theory and Applications, vol. 170, no. 1, pp. 266-293, 2016.

[18] D. Bauso and M. Cannon, "Consensus in opinion dynamics as a repeated game," Automatica, vol. 90, pp. 204-211, 2018.

[19] L. Smith and P. Sorensen, "Pathological outcomes of observational learning," Econometrica, vol. 68, no. 2, pp. 371-398, 2010.

[20] D. Acemoglu, M. A. Dahleh, I. Lobel, and A. Ozdaglar, "Bayesian learning in social networks," The Review of Economic Studies, vol. 78, no. 4, pp. 1201-1236, 2011.

[21] A. V. Banerjee, "A simple model of herd behavior," The Quarterly Journal of Economics, vol. 107, no. 3, pp. 797-817, 1992.

[22] L. J. Savage, The Foundations of Statistics, Dover Publications Inc, New York, NY, USA, 1972.

[23] S. Bikhchandani, D. Hirshleifer, and I. Welch, "A theory of fads, fashion, custom, and cultural change as informational cascades," Journal of Political Economy, vol. 100, no. 5, pp. 992-1026, 1992.

[24] B. M. G. Kibria, "Bayesian statistics and marketing," Technometrics, vol. 49, no. 2, p. 230, 2003.

[25] S. R. Gadre, "Some inequalities among expectation values of one-electron operators in atomic systems," The Journal of Chemical Physics, vol. 71, no. 3, p. 1510, 1979.

[26] W. W. Zachary, "An information flow model for conflict and fission in small groups," Journal of Anthropological Research, vol. 33, no. 4, pp. 452-473, 1977. 